\title{
Chronic lymphocytic thyroiditis (CLT) has a negative prognostic value in papillary thyroid cancer of the young and middle ages: a retrospective analysis of 635 patients
}

\section{Bing'e Ma}

Jiangsu Province Academy of Traditional Chinese Medicine https://orcid.org/0000-0002-4632-1542

\section{Xiyi Chen}

Nanjing Medical University

\section{Zhengping Zhao}

Jiangsu Province Academy of Traditional Chinese Medicine

Qin Ji

Nanjing University of Chinese Medicine

\section{Yifan Zhou}

Nanjing University of Chinese Medicine

Xiaoyang Yin

Nanjing University of Chinese Medicine

Chaoqun Ma ( $\sim$ dr_machaoqun@163.com )

Jianhua Wang

Jiangsu Province Academy of Traditional Chinese Medicine

\section{Research article}

Keywords: Papillary thyroid cancer, Chronic lymphocytic thyroiditis, Extrathyroidal extension, Recurrence risks, Age stratification

Posted Date: March 26th, 2020

DOI: https://doi.org/10.21203/rs.3.rs-18952/v1

License: @ (i) This work is licensed under a Creative Commons Attribution 4.0 International License. Read Full License 


\section{Abstract}

Background: The study was aimed at investigating the potential role of chronic lymphocytic thyroiditis (CLT) in papillary thyroid cancer (PTC) prognosis in distinct age groups, as well as the association between CLT and recurrence risk estimation.

Methods: A total of 635 adult patients were retrospectively analyzed. On the basis of postoperative pathology examination, 188 patients were diagnosed with coexistent CLT and the remaining 447 were classified as non-CLT. Then the characteristics of CLT-coexisted patients and non-CLT ones were compared respectively when patients were aged $\geq 55$ years or below. The association among postoperative clinicopathological features were also analyzed using multivariate regression. In addition, the prognostic value of several variables relating to high-risk recurrence were estimated within different age groups.

Results: When divided in two age groups (55 years as the line), non-CLT group had a remarkable frequency of small size lesion ( $\mathrm{m} \max \leq 1 \mathrm{~cm}$ ) compared with CLT-coexisted patients ( $54.6 \%$ to $43.0 \%, p=0.016)$. In addition, non-CLT patients tended to have intrathyroidal extension as opposed to those with coexistent CLT (20.2\% to $28.2 \%, p=0.05)$. In multivariate analysis, CLT still significantly acted as an independent risk factor of greater lesion size $(\mathrm{D}$ min $>1 \mathrm{~cm})(\mathrm{OR}=1.7, \mathrm{p}=0.02)$ and mildly promoted gross extrathyroidal extension $(E T E)(O R=1.4, p$ $=0.06)$. However, associations didn't emerge in the characteristics mentioned above with CLT when patients were $\geq 55$ years old. The prognostic value of CLT in high-risk recurrence was evident only in patients aged 35-44 years. $(\mathrm{OR}=2.4,95 \% \mathrm{Cl}: 1.2-5.4, \mathrm{p}=0.02)$. Greater lesion size independently promoted gross ETE, no matter patients were aged above 55 years or not. Besides, its prognostic value of high-risk recurrence was significant throughout all age groups.

Conclusion: These findings revealed that CLT coexistence might be the unfavorable factor of PTC prognosis in young and middle-aged ( $<55$ years) patients, and its role in recurrence risk stratification was presented only in the specific age (35-44 years).

\section{Introduction}

The most common histologic type of thyroid malignancy is papillary thyroid cancer (PTC), which accounts for about $90 \%$ of all thyroid malignant carcinomas (1). It has been reported more frequently than ever due to the accuracy and convenience of diagnostic technology such as ultrasound and fine-needle aspiration, which leads mainly to the increase of overall thyroid cancer incidence (2). Such relatively "benign" carcinoma with low mortality in the majority of cases has been considered to receive overtreatment for such a long time. However, poor prognosis can still remain when extrathyroidal extension or evident metastasis occurs (3).

Chronic lymphocytic thyroiditis (CLT) is an autoimmune disease histopathologically characterized by diffused infiltration of autoreactive $T$ and B cells (diffused lymphocyte infiltration, CLT) (4). Since it was first described by Dailey et al. (5) in 1955, a large amount of studies have carried out the controversial relationship between PTC and CLT (6-8). Considering the subclinical course in both disease and incidental diagnosis sometimes, conflicting data and conclusions are undoubtedly raised. It has been reported that CLT serves as an important role in the development of PTC (9) and increases the risk of PTC incidence $(10,11)$. Of note however, there still exists the advocation that CLT is associated with less aggressive clinicopathological presentation and a better 
prognosis of PTC $(12,13)$. In contrast, some reports also argued that CLT has no effect, or even negative effect, on the outcome of PTC $(14,15)$.

Besides, staging system for assessing PTC recurrence, which is one of the major concerns regarding treatments, has raised great attention $(16,17)$. Specifically, in terms of the association between CLT and PTC recurrence, many studies have suggested the strong link of coexistent CLT with a lower risk of recurrence $(12,18-20)$. However, the definition of "recurrence" in some of these studies actually referred to "persistence" because former "disease free" condition was not confirmed (usually refers to the absence of thyroglobulin or antithyroid globulin antibodies (21-23)). Recurrence is defined as the positive scan imaging of tumors (structural recurrence) after disease-free conditions (21-23). In that case, a predicting model of different recurrence risks was in need. According to the 2015 American Thyroid Association (ATA) Management Guidelines, three-tiered stratification system of structural recurrence risks was highly recommended for PTC patients after initial thyroidectomy, which was based on presence of several clinicopathologic features, including gross extrathyroidal extension and numbers of involved lymph nodes (24). These characteristics have also been observed due to the need to ensure their associations with coexistent CLT in PTC patients.

Herein, combined with the background mentioned above, our retrospective study was conducted to analyze the differences in clinicopathologic characteristics between adult PTC patients ( $\geq 18$ years) with coexistent CLT or not, as well as the potential role of CLT in estimating high-risk recurrence in different age groups.

\section{Materials And Methods}

Eligibility criteria and patient background

The retrospective analysis starts in December 2019, which collects 635 adult patients diagnosed with papillary thyroid cancer between February 2014 and December 2017 from all those 654 people confirmed as thyroid cancers, no matter what types, by histopathological examinations in the pathology department of Jiangsu Province Hospital on Integration of Chinese and Western Medicine. For the whole studied group, only patients diagnosed with PTC $(n=635)$ were enrolled, leaving other types excluded. All clinical features, including age, sex, preoperative ultrasound results and pathologic examinations were reviewed and confirmed according to previous electronic records. All patients underwent thyroidectomy or lobectomy for the first time.

Patients suspicious for malignancy in preoperative fine-needle aspiration biopsy or for lymph node metastases in imaging examinations all underwent unilateral lobectomy and prophylactic (operative) ipsilateral central lymph node dissection. If tumors were discovered invading capsules in the surgery, the total (or subtotal) thyroidectomy would be done. Lateral neck dissections would be performed if lateral lymph node metastases were suspected according to preoperative imaging evaluation. All the operations were excellently accomplished by an experienced surgeon who has been engaged in thyroid surgery for over two decades, and the postoperative biopsy was examined and reviewed by two seasoned pathologists.

Histopathological examinations

CLT was histopathologically defined as the presence of mononuclear lymphocytes infiltration in thyroid parenchyma and stroma, as well as a few reactive germinal centers with lymphoid follicle formation, parenchymal atrophy and abundant oxyphilic cell changes in follicular cells (25-27). Multifocality means that at 
least one lobe was discovered with above one lesion existing. Lesion size stratification was divided as maximum diameter $\leq 1 \mathrm{~cm}$ or minimum lesion diameter above $1 \mathrm{~cm}$.

\section{Postoperative stratification}

Assessment of each patient's clinical stage was according to the 8th edition of TNM system (2016), for which the histopathology associated classification is reestimated on the basis of pathology reports. In addition, MACIS score is used for differentiated thyroid cancer, and the calculation was based on several indexes, including age, tumor size, whether with resecting operation, local invasiveness or distant metastasis (28). Gender, age, preoperative CLT, as well as pathologic evidence of tumor size, central lymph node metastasis (CLNM), lateral lymph node metastasis (LLNM), multifocality, TNM staging and gross extrathyroidal extension after surgery were recorded for each patient involved. Gross extrathyroidal extension (ETE) refers to the gross soft-tissue invasion identified on clinical examination, intraoperatively or on imaging, which conveys an increased risk of mortality. The newly modified clinicopathologic recurrence risk stratification system was proposed in the 2015 version of the American Thyroid Association (ATA) thyroid cancer guidelines, and it was used to classify patients as low-, intermediate- or high-risk recurrence (24). In our study, patients confirmed with gross ETE were all classified as having high-risk recurrence. Those with absence of gross ETE but diagnosed with $>5$ involved pathologic LN metastasis, of which the largest dimension was $<3 \mathrm{~cm}$, were classified in intermediate-risk recurrence. Otherwise, the patients would be classified in low-risk recurrent disease, who were characterized with no local or distant metastases, and with absence of locoregional invasion. Our five age groups were stratified as 18-24, 25-34, 35$44,45-54$ and $\geq 55$ years, which was applied to analyzing the role of CLT predicting risks of recurrence.

\section{Statistical analysis}

All data processing was undertaken with IBM SPSS Statistics for MacOS version 23.0.

The Pearson's Chi Square test and contingency tables were used to compare categorical variables. The comparisons between non-normally distributed variables were presented by Mann-Whitney U test. Otherwise, the independent t-test would be utilized for distributed ones. Binary logistic regression analysis was applied to estimate odds ratio (OR) for dichotomous dependent variables. For ordered dependent variables, ordinal regression analysis was in use. Besides, we employed the collinearity diagnostics to exclude collinearity problems. Results showed that variance inflation factor (VIF) was far below 10 and tolerance was higher than 0.1 between variables in the multiple regression model. No collinearity existed among sex, age, CLT and relevant dependent variables in respective regression analysis. $P$ value $<0.05$ was considered statistically significant.

\section{Results}

Basic Clinicopathological features in all patients

Among the 635 PTC patients evaluated in the study, 188 (29.6\%) had coexisting CLT while the remaining 447 (70.1\%) were found to be non-CLT. The proportion of female in non-CLT and CLT-coexisted group was 70.5\% and 89.9\% ( $p<0.001)$, with mean ages being $43.6 \pm 11.9$ and $44.1 \pm 12.3(p=0.65)$ for each. A total of 325 patients were diagnosed as small lesion (with largest lesion diameter $\leq 1 \mathrm{~cm}$ ) and 143 patients were confirmed with gross extrathyroidal extension (ETE). 623 patients were classified in TNM stage I, leaving only 12 patients in stage II-IV (Table 1). 
Table 1

Clinicopathological characteristics of non-CLT and CLT-coexisted groups in all patients involved

\begin{tabular}{|c|c|c|c|c|c|}
\hline Characteristics & Total & non-CLT(\%) & CLT-coexisted(\%) & OR(95\%Cl) & $P$ value \\
\hline Number of patients & 635 & $447(70.1)$ & $188(29.6)$ & & \\
\hline \multicolumn{6}{|l|}{ Gender } \\
\hline Female & 484 & $315(70.5)$ & $169(89.9)$ & $3.7(2.2-6.2)$ & $<0.001$ \\
\hline Male & 151 & $132(29.5)$ & $19(10.1)$ & & \\
\hline \multicolumn{6}{|l|}{ Age } \\
\hline$<55$ & 515 & $366(71.1)$ & $149(28.9)$ & $1.1(0.8-1.6)$ & 0.49 \\
\hline$\geq 55$ & 382 & $81(67.5)$ & $39(32.5)$ & & \\
\hline Small lesion (Dmax $\leq 1 \mathrm{~cm})$ & 325 & $240(53.7)$ & $85(45.2)$ & $1.4(1.0-2.0)$ & 0.05 \\
\hline Extrathyroidal extension & 143 & $94(21.0)$ & $49(26.1)$ & $1.3(0.9-2.0)$ & 0.17 \\
\hline CLNM & 297 & $219(49.0)$ & $78(41.5)$ & $0.7(0.5-1.0)$ & 0.08 \\
\hline LLNM & 54 & $40(8.9)$ & $14(7.4)$ & $0.8(0.4-1.5)$ & 0.54 \\
\hline \multicolumn{6}{|l|}{ TNM stage } \\
\hline ૫ & 623 & $438(98.0)$ & $185(98.4)$ & $0.8(0.2-2.9)$ & 0.72 \\
\hline$\nabla-\nabla$ & 12 & $9(2.0)$ & $3(1.6)$ & & \\
\hline Multifocality & 194 & $133(29.8)$ & $61(33.0)$ & $1.2(0.8-1.7)$ & 0.43 \\
\hline \multicolumn{6}{|l|}{ MACIS score } \\
\hline$<6$ & 595 & 417 (93.3) & $178(94.7)$ & $1.3(0.6-2.7)$ & 0.51 \\
\hline $6-6.99$ & 31 & $24(5.4)$ & $7(3.7)$ & $0.7(0.3-1.6)$ & 0.38 \\
\hline $7-7.99$ & 9 & $6(1.3)$ & $3(1.6)$ & $1.2(0.3-4.8)$ & 0.81 \\
\hline High-risk recurrence & 143 & $94(21.0)$ & $49(26.1)$ & $1.3(0.9-2.0)$ & 0.17 \\
\hline \multicolumn{6}{|c|}{${ }^{*}$ CLNM: central lymph node metastasis } \\
\hline $\begin{array}{l}\text { LLNM: lateral lymph node me } \\
\text { OR: odds ratio }\end{array}$ & stasis & & & & \\
\hline
\end{tabular}

CLT and features of PTC

With regard to the presence of extrathyroidal extension, there were 49 cases in CLT-coexisted group and 94 (26.1$21.0 \%, p=0.166)$ in the non-CLT one. In addition, the non-CLT group seemed to have a greater portion of smaller lesion size than the CLT-coexisted group (53.7-45.2\%, $\mathrm{p}=0.05)$, and there was a trend to have central LN metastasis in patients without coexistent CLT, although the difference didn't reach set significance $(49.0 \%$ to $41.5 \%, p=0.08)$. No evident differences were shown in high-risk recurrence stratification between two groups ( $p=$ 0.17). There was no statistically significant difference in multifocality, TNM staging or MACIS score (Table 1). 
A total of 515 patients were aged < 55 years, of whom 149 (28.9\%) were diagnosed with coexisting CLT and the other 366 (71.1\%) without. CLT-coexisted group had more female patients than the CLT-coexisted one (89.3$68.3 \%, p<0.001)$. There seemed to be a greater portion of patients diagnosed as small lesion in non-CLT group compared with the CLT-coexisted group $(54.6-43.0 \%, p=0.02)$. The gross ETE was found more prevalent in CLTcoexisted group than the non-CLT one $(28.2-20.2 \%, p=0.04)$. There wasn't any significant difference shown in CLNM $(55.2-47.7 \%, p=0.12)$ or LLNM $(10.7-9.4 \%, p=0.67)$ presence between non-CLT patients and patients coexisted with CLT (Table 2).

In all the 120 patients aged $\geq 55$ years, 81 were classified as non-CLT group and the other 39 as CLT-coexisted one (67.5-32.5\%). The difference in gender proportion between the two groups didn't reach significance. In addition, no significant difference was shown in lesion size, gross ETE, CLNM or LLNM between the two groups (Table 3).

In patients aged $<55$ years, $\operatorname{CLT}(O R=1.7, p=0.02)$ served as the independent risk factor of greater lesion size in multivariate analysis. Female patients were more likely to have small lesions $(O R=0.6, p=0.04)$. In addition, gross ETE was evidently associated with the larger lesion $(O R=3.3, p<0.001)$ in multivariate analysis. In terms of macroscopic ETE, CLT coexistence promoted the risk of ETE $(O R=1.4, p=0.06)$ according to the multivariate analysis, though the trend didn't reach significance. The greater lesion size also independently predicted ETE presence in return $(\mathrm{OR}=3.3, \mathrm{p}<0.001)$. There wasn't any significant difference in CLNM or LLNM between CLTcoexisted patients and those without, and CLT didn't cast effect on CLNM ( $p=0.19)$. The role of gross ETE took the positive influence on both CLNM $(O R=2.4, p<0.001)$ and LLNM $(O R=3.1, p<0.001)$ presence, respectively. Greater lesion size also positively affected CLNM $(O R=1.8, p=0.002)$ and LLNM $(O R=4.5, p<0.001)(T a b l e ~ 4)$.

For patients $\geq 55$ years, only the greater lesion size worked as an independent factor of gross ETE and CLNM (Table 5). 
Table 5

Association among different clinicopathological characteristics in patients $\geq 55$ years

\begin{tabular}{|c|c|c|c|c|c|}
\hline Characteristics & Total & non-CLT(\%) & $\begin{array}{l}\text { CLT- } \\
\text { coexisted(\%) }\end{array}$ & $\mathrm{OR}(95 \% \mathrm{Cl})$ & $\begin{array}{l}P \\
\text { value }\end{array}$ \\
\hline Number of patients & 515 & $366(71.1)$ & $149(28.9)$ & & \\
\hline \multicolumn{6}{|l|}{ Gender } \\
\hline Female & 383 & $250(68.3)$ & $133(89.3)$ & $3.9(2.2-6.8)$ & $\begin{array}{l}<.001 \\
0.001\end{array}$ \\
\hline Male & 132 & $116(31.7)$ & $16(10.7)$ & & \\
\hline Small lesion (Dmax $\leq 1 \mathrm{~cm})$ & 264 & $200(54.6)$ & $64(43.0)$ & $1.6(1.2-2.4)$ & 0.02 \\
\hline Extrathyroidal extension & 116 & $74(20.2)$ & $42(28.2)$ & $1.5(1.1-2.4)$ & 0.04 \\
\hline CLNM & 273 & $202(55.2)$ & $71(47.7)$ & $0.7(0.5-1.1)$ & 0.12 \\
\hline LLNM & 53 & $39(10.7)$ & $14(9.4)$ & $0.9(0.5-1.7)$ & 0.67 \\
\hline \multicolumn{6}{|l|}{ TNM stage } \\
\hline$\square$ & 515 & $366(71.1)$ & 149 (28.9) & & \\
\hline$\nabla-\varnothing$ & 0 & & & & \\
\hline Multifocality & 152 & $102(27.9)$ & $50(34.0)$ & $1.3(0.9-2.0)$ & 0.27 \\
\hline \multicolumn{6}{|c|}{ * CLNM: central lymph node metastasis } \\
\hline \multicolumn{6}{|c|}{ LLNM: lateral lymph node metastasis } \\
\hline OR: odds ratio & & & & & \\
\hline
\end{tabular}

* ETE: extrathyroidal extension

CLNM: central lymph node metastasis

OR: odds ratio 
Table 2 Association between CLT and clinicopathological characteristics in patients $<55$ years

Table 3 Association between CLT and clinicopathological characteristics in patients $\geq 55$ years

\begin{tabular}{|c|c|c|c|c|c|}
\hline Charateristics & Total & non-CLT(\%) & CLT-coexisted(\%) & $\mathrm{OR}(95 \% \mathrm{Cl})$ & $P$ value \\
\hline Number of patients & 120 & $81(67.5)$ & $39(32.5)$ & & \\
\hline \multicolumn{6}{|l|}{ Gender } \\
\hline Female & 101 & $65(80.2)$ & $36(92.3)$ & $3.0(0.8-10.8)$ & 0.09 \\
\hline Male & 19 & $16(19.8)$ & $3(7.7)$ & & \\
\hline Small lesion (Dmax $\leq 1 \mathrm{~cm})$ & 61 & $40(49.4)$ & $21(53.8)$ & $0.8(0.4-1.8)$ & 0.29 \\
\hline Extrathyroidal extension & 27 & $20(24.7)$ & $7(17.9)$ & $0.7(0.3-1.7)$ & 0.41 \\
\hline CLNM & 24 & $17(21.0)$ & 7 (17.9) & $0.8(0.3-2.2)$ & 0.7 \\
\hline LLNM & 1 & $1(1.2)$ & 0 & $1.0(0.9-1.0)$ & 0.49 \\
\hline \multicolumn{6}{|l|}{ TNM stage } \\
\hline प & 108 & $72(88.9)$ & $36(92.3)$ & $0.7(0.2-2.6)$ & 0.56 \\
\hline$\bigotimes-\square$ & 12 & $9(11.1)$ & $3(7.7)$ & & \\
\hline Multifocality & 42 & $31(38.3)$ & $11(28.9)$ & $0.7(0.3-1.5)$ & 0.32 \\
\hline \multicolumn{6}{|c|}{ * CLNM: central lymph node metastasis } \\
\hline \multicolumn{6}{|c|}{ LLNM: lateral lymph node metastasis } \\
\hline OR: odds ratio & & & & & \\
\hline
\end{tabular}

Table 4 Association among different clinicopathological characteristics in patients $<55$ years

Characteristics Univariate OR P value Multivariate OR P value

Greater Size of

lesion

\begin{tabular}{|lllll|}
\hline Female & 0.7 & 0.08 & 0.6 & 0.04 \\
\hline Age & 0.9 & 0.006 & 0.9 & 0.02 \\
\hline CLT & 1.6 & 0.02 & 1.7 & 0.02 \\
Gross ETE & 3.5 & $<0.001$ & 3.3 & $<0.001$ \\
Gross ETE & & & & 0.68 \\
Female & 0.9 & 0.58 & 0.9 &
\end{tabular}

* ETE: extrathyroidal extension

CLNM: central lymph node metastasis

OR: odds ratio 
Table 2 Association between CLT and clinicopathological characteristics in patients $<55$ years

\begin{tabular}{|c|c|c|c|c|}
\hline Age & 1 & 0.16 & 1 & 0.48 \\
\hline CLT & 1.5 & 0.04 & 1.4 & 0.06 \\
\hline $\begin{array}{l}\text { Greater Size of } \\
\text { lesion }\end{array}$ & 3.5 & $<0.001$ & 3.3 & $<0.001$ \\
\hline \multicolumn{5}{|l|}{ CLNM } \\
\hline Female & 0.6 & 0.009 & 0.7 & 0.09 \\
\hline Age & 0.9 & $<0.001$ & 0.9 & $<0.001$ \\
\hline CLT & 0.7 & 0.12 & 0.7 & 0.06 \\
\hline $\begin{array}{l}\text { Greater Size of } \\
\text { lesion }\end{array}$ & 2.2 & $<0.001$ & 1.8 & 0.002 \\
\hline Gross ETE & 2.8 & $<0.001$ & 2.4 & $<0.001$ \\
\hline \multicolumn{5}{|l|}{ LLNM } \\
\hline Female & 1.2 & 0.6 & 1.6 & 0.19 \\
\hline Age & 0.9 & 0.22 & 1 & 0.59 \\
\hline CLT & 0.9 & 0.67 & 0.6 & 0.12 \\
\hline $\begin{array}{l}\text { Greater Size of } \\
\text { lesion }\end{array}$ & 5.3 & $<0.001$ & 4.5 & $<0.001$ \\
\hline Gross ETE & 4.0 & $<0.001$ & 3.1 & $<0.001$ \\
\hline
\end{tabular}

\begin{tabular}{|lllll} 
Characteristics & $\begin{array}{l}\text { Univariate } \\
\text { OR }\end{array}$ & $\begin{array}{l}\text { P } \\
\text { value }\end{array}$ & $\begin{array}{l}\text { Multivariate } \\
\text { OR }\end{array}$ & $\begin{array}{l}\mathbf{P} \\
\text { value }\end{array}$ \\
\hline $\begin{array}{l}\text { Greater Size of } \\
\text { lesion }\end{array}$ & & & & \\
\hline Female & 0.8 & 0.74 & 1.3 & 0.64 \\
\hline Age & 1.1 & 0.04 & 1.1 & 0.19 \\
\hline CLT & 0.8 & 0.65 & 0.9 & 0.81 \\
\hline Gross ETE & 6.7 & $<$ & 6.0 & 0.001 \\
\hline
\end{tabular}

* ETE: extrathyroidal extension

CLNM: central lymph node metastasis

OR: odds ratio 


\section{Table 2 Association between CLT and clinicopathological characteristics in patients $<55$ years}

\begin{tabular}{|lllll}
\hline Gross ETE & \multicolumn{5}{l}{} \\
\hline Female & 0.4 & 0.11 & 0.5 & 0.26 \\
\hline Age & 1.1 & 0.009 & 1.1 & 0.08 \\
\hline CLT & 0.7 & 0.41 & 0.7 & 0.56 \\
$\begin{array}{l}\text { Greater Size of } \\
\text { lesion }\end{array}$ & 6.7 & $<$ & 5.9 & 0.001 \\
\hline CLNM & & 0.001 & & \\
\hline Female & 0.9 & 0.9 & 1.1 & 0.85 \\
\hline Age & 1.1 & 0.29 & 1 & 0.79 \\
\hline * ETE: extrathyroidal extension & & \\
\hline CLNM: central lymph node metastasis & & \\
\hline
\end{tabular}

CLT's role in estimating high-risk recurrent disease was only shown in patients of 35-44 years

In our whole studied group, only the greater lesion size, instead of CLT, gave rise to high-risk recurrence in the multivariate analysis $(\mathrm{OR}=3.5,95 \% \mathrm{Cl} 2.4-5.0, \mathrm{p}<0.001)$. When classified in different age cohorts, the promotion still remained. CLT didn't significantly affect recurrence stratification in the whole studied group $(\mathrm{OR}=1.2,95 \% \mathrm{Cl}$ $0.8-1.8, p=0.31$ ). According to multivariate analysis in different age cohorts, CLT's positive effect on high-risk recurrence stratification was shown significant merely in patients aged between $35-44$ years $(O R=2.4,95 \% \mathrm{Cl}$ $1.1-5.4, p=0.02$ ). No other age cohorts existed evident effect of CLT in multivariate analysis (Table 6 , see Additional file 1).

\section{Discussion}

The unambiguous effect of coexisting CLT has always been under debate, due to conflicting data of CLT and risk of malignancy. The contradiction could be attributed to different definition of CLT, contradicted effects of CLT in different age groups, poor pathologic reports of coexistent CLT (29) or variation of statistical approaches. In this study, multivariate analysis was performed to indicate that histopathologically confirmed CLT was independently associated with several aggressive pathologic features in patients $<55$ years to some extent, which could suggest in a way that autoimmune thyroiditis harbored unfavorable impacts on PTC progress.

It has been indicated in previous abundant data that PTC coexistent with CLT was nearly three times more frequent than non-CLT (30). Our study has showed that $29.6 \%$ of PTC patients had coexistent CLT, which was a consistent frequency with statistics in other reports $(0.5-38 \%)(18,31-33)$. Meanwhile, our study also presented that coexistent CLT was more prevalent in female PTC patients, which was similar to conclusions in other reports (34). In our entire studied sample, coexistent CLT didn't show significant association with any aggressive features in malignancy, except that CLT-coexisted group had a mild trend to have lesions $>1 \mathrm{~cm}$. However, we found that in patients $<55$ years, those with coexistent CLT tended to have more extrathyroidal extension and significantly a 
less frequency of small lesions. While in patients $\geq 55$ years, the presence of CLT was associated with none of the clinical or pathologic features.

Our analyzed results indicated that CLT independently predicted macroscopic ETE and larger lesions (minimum lesion size $>1 \mathrm{~cm}$ ) in patients $<55$ years, and this trend was consistent with several previous reports. Babli et al. (25) concluded that the presence of CLT could be in association with unfavorable pathologic features, including ETE in young patients. Nam et al. (26) observed the larger lesions in CLT-coexisted patients. In contrast, however, CLT played a protective role in cancer development concluded from some other investigations. According to the study from Kim et al. (27), CLT was an independent predictor for low frequency of ETE and CLNM. We observed that the greater lesion size $\left(D_{\text {min }}>1 \mathrm{~cm}\right)$ served as an independent risk factor of gross ETE in patients and was associated with high-risk recurrence stratification in all age cohorts. Besides, ETE and greater lesion size mutually promoted the progression of each other, which had also been supported by other investigations that, according to Kim et al. (27), greater tumor size was independently associated with gross ETE, and vice versa. According to Castro et al. (35), larger lesion size was one of the best predictors of persistence/recurrence. Our observation was in accordance with studies from Lee et al. (36), that larger tumor size was one of the independent risk factors of gross ETE, which could partly explain the unfavorable prognosis. Apart from the authoritative statement in ATA guideline, gross ETE has been a longtime recognized adverse pathologic predictive factor of worse outcome in many other studies, and the extent of macroscopic ETE appeared to be a potential determinant of PTC recurrence. The macroscopic (gross) extrathyroidal extension has been proposed to be a well-established key variable and of more importance in PTC prognosis stratifications, compared with microscopic ETE (37). However, it has to be underlined that pathological and clinical features in 2015 ATA risk stratification systems are often diagnosed subjectively and vary in different institutions, so the definite approach to estimating high-risk recurrence still needs further observation so that it can play a part in bettering clinical management.

The results of CLT's role in initial recurrence risk estimation from the whole studied group didn't show significance. Interestingly, when divided in different age groups, CLT showed evident prognosis value but only in patients aged 35-44 years. No previous investigation has demonstrated CLT's role in recurrence risk stratification, especially in different age groups respectively. In that case, our observation could give a hint in regard to subsequent clinical management, of which the focus could be placed on patients in middle age.

In fact, the association between CLT and PTC outcome has remained controversy for over two decades. It is still ambiguous and unexplained partly because of the hesitation in answering whether it is the concurrence or causality between them. One hypothesis elucidated in previous studies pointed out that the lymphocytes migration around tumor lesions (known as "peritumoral infiltration") was trying to restrict malignancy progression (38). This phenomenon, however, is distinct from the diffused lymphocyte infiltration in CLT with follicle effacement. Kamma et al. (39) observed that lymphocyte infiltration, predominantly around the tumor, was correlated with more aggressive cases. They supposed that surrounding lymphocyte infiltration was followingly induced by the antigen expression on tumor cells, which aroused the necessary but insufficient immune attack. Other hypotheses were addressed in different ways that the coexisting CLT contributed to cancer progression. Notably, thyroid follicular epithelial cells can show oncocytic changes extensively, exhibiting crowding, irregular contours and large nucleus, which resembled features of papillary carcinoma (40). According to Giordano et al. (41), the follicular cells in CLT background intensely expressed Fas/FasL due to abundant interleukin-1 beta (IL$1 \beta \mathrm{d})$, which activated the apoptosis pathway to cause the destruction of normal thyroid tissue, promoting 
carcinoma growth and malignancy. Despite the abundant reports with regard to the association between CLT and PTC, the exact mechanism is still under debate and in need of further research.

The limitations of our study should be considered that it is a retrospective study, and CLT diagnosis was confirmed on the basis of histopathological examination on thyroid tissue from thyroidectomy, which concealed the real chronological and further causal relationship between PTC and CLT. Besides, we simply estimate recurrence risks using initial postoperative stratification system, the robust association between CLT and recurrence needs factual prospective outcomes. The significant results were only applied to patients $<55$ years and the prognosis value of CLT was only apparent in patients aged in 35-44 years, so the finer investigation towards age specificity and possible mechanisms should be conducted in the study hindward.

In accordance with previous reports, CLT coexistence was demonstrated much more prevalent in female. We conclude that CLT served as an independent risk factor of some aggressive clinical characteristics, including gross extrathyroidal extension and greater lesion size $(>1 \mathrm{~cm})$ in PTC patients with coexistent CLT of younger age (<55 years). In the light of authoritative stratification from ATA guideline, PTC patients aged in 35-44 years were estimated more likely to undergo high-risk recurrence.

\section{Abbreviations}

PTC: papillary thyroid cancer; CLT: chronic lymphocytic thyroiditis; ATA: American Thyroid Association; CLNM: central lymph node metastasis; LLNM: lateral lymph node metastasis; ETE: extrathyroidal extension

\section{Declarations}

Ethics approval and consent to participate

The study was approved by the institutional review board of Jiangsu Province Hospital on Integration of Chinese and Western Medicine, Nanjing, Jiangsu Province, China and the study was performed in accordance with the Declaration of Helsinki and Good Clinical Practice guidelines (IRB number: 2019LWKY006). The requirement for studied clinicopathologic features of each patient involved has been informed and consented.

Consent for publication

Not applicable.

Availability of data and materials

The data analyzed that support the findings of this study are available from the corresponding author upon reasonable request.

Competing interests

Page 12/16 
The authors declare that they have no competing interests.

Funding

This work was supported by Jiangsu Province Health Commission (Grant Number: BJ18029). The funding sources took no role in the design of the study; in the collection, analysis, and interpretation of data; or in the writing of the manuscript.

Authors' contributions

MBE and CXY: carried out the studies, participated in collecting data, designed and drafted the manuscript. ZZP: performed the statistical analysis. YXY, JQ and ZYF: participated in collecting data. MCQ and WJH: reviewed and edited the manuscript. All authors read and approved the final manuscript.

Acknowledgements

Not applicable.

Author details

${ }^{1}$ Department of Thyroid and Breast Surgery, Affiliated Hospital of Integrated Traditional Chinese and Western Medicine for Nanjing University of Chinese Medicine, Nanjing 210029, China. ${ }^{2}$ The First School of Clinical Medicine, Nanjing Medical University, Nanjing 211166, China. ${ }^{3}$ The First School of Clinical Medicine, Nanjing University of Chinese Medicine, Nanjing 210023, China. ${ }^{4}$ Department of General Surgery, Affiliated Hospital of Nanjing University of Chinese medicine, Nanjing 210029, China

\section{References}

1. Huang Y, Qu S, Zhu G, Wang F, Liu R, Shen X, et al. BRAF V600E Mutation-Assisted Risk Stratification of Solitary Intrathyroidal Papillary Thyroid Cancer for Precision Treatment. JNCl: Journal of the National Cancer Institute. 2018;110(4):362-70.

2. Zhao H, Li H, Huang T. High Urinary lodine, Thyroid Autoantibodies, and Thyroid-Stimulating Hormone for Papillary Thyroid Cancer Risk. Biol Trace Elem Res. 2018;184(2):317-24.

3. Takano T. Natural history of thyroid cancer [Review]. Endocr J. 2017;64(3):237-44.

4. Ahmed R, Al-Shaikh S, Akhtar M. Hashimoto thyroiditis: a century later. Adv Anat Pathol. 2012;19(3):181-6. 
5. DAILEY ME, LINDSAY S, SKAHEN R. Relation of thyroid neoplasms to Hashimoto disease of the thyroid gland. AMA Arch Surg. 1955;70(2):291-7.

6. Shen C-T, Zhang X-Y, Qiu Z-L, Sun Z-K, Wei W-J, Song H-J, et al. Thyroid autoimmune antibodies in patients with papillary thyroid carcinoma: a double-edged sword? Endocrine. 2017;58(1):176-83.

7. Dvorkin S, Robenshtok E, Hirsch D, Strenov Y, Shimon I, Benbassat CA. Differentiated thyroid cancer is associated with less aggressive disease and better outcome in patients with coexisting Hashimotos thyroiditis. J Clin Endocrinol Metab. 2013;98(6):2409-14.

8. Kwak HY, Chae BJ, Eom YH, Hong YR, Seo JB, Lee SH, et al. Does papillary thyroid carcinoma have a better prognosis with or without Hashimoto thyroiditis? Int J Clin Oncol. 2015;20(3):463-73.

9. Song E, Oh H-S, Jeon MJ, Chung KW, Hong SJ, Ryu JS, et al. The value of preoperative antithyroidperoxidase antibody as a novel predictor of recurrence in papillary thyroid carcinoma. Int J Cancer. 2019;144(6):1414-20.

10. Lun Y, Wu X, Xia Q, Han Y, Zhang X, Liu Z, et al. Hashimoto's thyroiditis as a risk factor of papillary thyroid cancer may improve cancer prognosis. Otolaryngol Head Neck Surg. 2013;148(3):396-402.

11. Repplinger D, Bargren A, Zhang Y-W, Adler JT, Haymart M, Chen H. Is Hashimoto's thyroiditis a risk factor for papillary thyroid cancer? J Surg Res. 2008;150(1):49-52.

12. Kebebew E, Treseler PA, Ituarte $\mathrm{PH}$, Clark $\mathrm{OH}$. Coexisting chronic lymphocytic thyroiditis and papillary thyroid cancer revisited. World J Surg. 2001;25(5):632-7.

13. Huang B-Y, Hseuh C, Chao T-C, Lin K-J, Lin J-D. Well-differentiated thyroid carcinoma with concomitant Hashimoto's thyroiditis present with less aggressive clinical stage and low recurrence. Endocr Pathol. 2011;22(3):144-9.

14. Del Rio P, Cataldo S, Sommaruga L, Concione L, Arcuri MF, Sianesi M. The association between papillary carcinoma and chronic lymphocytic thyroiditis: does it modify the prognosis of cancer? Minerva Endocrinol. 2008;33(1):1-5.

15. Konturek A, Barczyński M, Wierzchowski W, Stopa M, Nowak W. Coexistence of papillary thyroid cancer with Hashimoto thyroiditis. Langenbecks Arch Surg. 2013;398(3):389-94.

16. Ito Y, Kudo T, Kobayashi K, Miya A, Ichihara K, Miyauchi A. Prognostic factors for recurrence of papillary thyroid carcinoma in the lymph nodes, lung, and bone: analysis of 5,768 patients with average 10-year follow-up. World J Surg. 2012;36(6):1274-8.

17. Sanders LE, Cady B. Differentiated thyroid cancer: reexamination of risk groups and outcome of treatment. Arch Surg. 1998;133(4):419-25.

18. Kashima K, Yokoyama S, Noguchi S, Murakami N, Yamashita H, Watanabe S, et al. Chronic thyroiditis as a favorable prognostic factor in papillary thyroid carcinoma. Thyroid. 1998;8(3):197-202.

19. Loh KC, Greenspan FS, Dong F, Miller TR, Yeo PP. Influence of lymphocytic thyroiditis on the prognostic outcome of patients with papillary thyroid carcinoma. J Clin Endocrinol Metab. 1999;84(2):458-63.

20. Jeong JS, Kim HK, Lee C-R, Park S, Park JH, Kang S-W, et al. Coexistence of chronic lymphocytic thyroiditis with papillary thyroid carcinoma: clinical manifestation and prognostic outcome. J Korean Med Sci. 2012;27(8):883-9.

21. Tuttle RM, Tala H, Shah J, Leboeuf R, Ghossein R, Gonen M, et al. Estimating risk of recurrence in differentiated thyroid cancer after total thyroidectomy and radioactive iodine remnant ablation: using 
response to therapy variables to modify the initial risk estimates predicted by the new American Thyroid Association staging system. Thyroid. 2010;20(12):1341-9.

22. Castagna MG, Maino F, Cipri C, Belardini V, Theodoropoulou A, Cevenini G, et al. Delayed risk stratification, to include the response to initial treatment (surgery and radioiodine ablation), has better outcome predictivity in differentiated thyroid cancer patients. Eur J Endocrinol. 2011;165(3):441-6.

23. Jeon MJ, Kim WG, Park WR, Han JM, Kim TY, Song DE, et al. Modified dynamic risk stratification for predicting recurrence using the response to initial therapy in patients with differentiated thyroid carcinoma. Eur J Endocrinol. 2014;170(1):23-30.

24. Haugen BR. 2015 American Thyroid Association Management Guidelines for Adult Patients with Thyroid Nodules and Differentiated Thyroid Cancer: What is new and what has changed? Cancer. 2017;123(3):37281.

25. Babli S, Payne RJ, Mitmaker E, Rivera J. Effects of Chronic Lymphocytic Thyroiditis on the Clinicopathological Features of Papillary Thyroid Cancer. Eur Thyroid J. 2018;7(2).

26. Nam HY, Lee HY, Park GC. Impact of co-existent thyroiditis on clinical outcome in papillary thyroid carcinoma with high preoperative serum antithyroglobulin antibody: a retrospective cohort study. Clin Otolaryngol. 2016;41(4):358-64.

27. Kim SK, Woo J-W, Lee JH, Park I, Choe J-H, Kim J-H, et al. Chronic lymphocytic thyroiditis and BRAF V600E in papillary thyroid carcinoma. Endocr Relat Cancer. 2016;23(1):27-34.

28. Hay ID, Bergstralh EJ, Goellner JR, Ebersold JR, Grant CS. Predicting outcome in papillary thyroid carcinoma: development of a reliable prognostic scoring system in a cohort of 1779 patients surgically treated at one institution during 1940 through 1989. Surgery. 1993;114(6).

29. Meier DW, Woolner LB, Beahrs OH, McConahey WM. Parenchymal findings in thyroidal carcinoma: pathologic study of 256 cases. J Clin Endocrinol Metab. 1959;19(1):162-71.

30. Lee J-H, Kim Y, Choi J-W, Kim Y-S. The association between papillary thyroid carcinoma and histologically proven Hashimoto's thyroiditis: a meta-analysis. Eur J Endocrinol. 2013;168(3):343-9.

31. Cipolla C, Sandonato L, Graceffa G, Fricano S, Torcivia A, Vieni S, et al. Hashimoto thyroiditis coexistent with papillary thyroid carcinoma. Am Surg. 2005;71(10):874-8.

32. Matesa-Anić D, Matesa N, Dabelić N, Kusić Z. Coexistence of papillary carcinoma and Hashimoto's thyroiditis. Acta Clin Croat. 2009;48(1).

33. Braverman LE. lodine and the thyroid: 33 years of study. Thyroid. 1994;4(3):351-6.

34. Liang J, Zeng W, Fang F, Yu T, Zhao Y, Fan X, et al. Clinical analysis of Hashimoto thyroiditis coexistent with papillary thyroid cancer in 1392 patients. Acta Otorhinolaryngol Ital. 2017;37(5):393-400.

35. de Castro TP, Waissmann W, Simões TC, de Mello RCR, Carvalho DP. Predictors for papillary thyroid cancer persistence and recurrence: a retrospective analysis with a 10-year follow-up cohort study. Clin endocrinol. 2016;85(3):466-74.

36. Lee I, Kim HK, Soh EY, Lee J. The Association Between Chronic Lymphocytic Thyroiditis and the Progress of Papillary Thyroid Cancer. World J Surg. 2020.

37. Abraham E, Roshan D, Tran B, Wykes J, Campbell P, Ebrahimi A. The extent of extrathyroidal extension is a key determinant of prognosis in T4a papillary thyroid cancer. J Surg Oncol. 2019;120(6):1016-22.

Page 15/16 
38. Fridman WH, Galon J, Pagès F, Tartour E, Sautès-Fridman C, Kroemer G. Prognostic and predictive impact of intra- and peritumoral immune infiltrates. Cancer Res. 2011;71(17):5601-5.

39. Kamma H, Fujii K, Ogata T. Lymphocytic infiltration in juvenile thyroid carcinoma. Cancer. 1988;62(9):198893.

40. Asa SL. My approach to oncocytic tumours of the thyroid. J Clin Pathol. 2004;57(3):225-32.

41. Giordano C, Stassi G, De Maria R, Todaro M, Richiusa P, Papoff G, et al. Potential involvement of Fas and its ligand in the pathogenesis of Hashimoto's thyroiditis. Science (New York, NY). 1997;275(5302):960-3.

\section{Supplementary Files}

This is a list of supplementary files associated with this preprint. Click to download.

- Additionalfile1Table6.xlsx 